\title{
Les reliques des saints. Publications récentes et perspectives
} nouvelles (II)

\section{Philippe George}

Citer ce document / Cite this document :

George Philippe. Les reliques des saints. Publications récentes et perspectives nouvelles (II). In: Revue belge de philologie et d'histoire, tome 82, fasc. 4, 2004. Histoire médiévale, moderne et contemporaine - Middeleeuwse. moderne en hedendaagse geschiedenis. pp. 1041-1056;

doi : https://doi.org/10.3406/rbph.2004.4877

https://www.persee.fr/doc/rbph_0035-0818_2004_num_82_4_4877

Fichier pdf généré le 17/04/2018 


\title{
BIBLIOGRAPHIE - BIBLIOGRAFIE
}

\section{Les reliques des saints. Publications récentes et perspectives nouvelles (II)}

\author{
Philippe GEORGE \\ Trésor de la Cathédrale de Liège
}

Le culte des reliques imprègne considérablement la mentalité médiévale: on a beau le répéter, on peut encore être surpris de ses implications au fil des nouvelles recherches $\left({ }^{1}\right)$. Ainsi, dans son imposant Origins of the European Economy, Michael Mc Cormick consacre un chapitre aux Hagiographical Horizons: Collecting Exotic Relics in Early Medieval France ${ }^{2}$ ).

Les débuts du culte des reliques en Occident ont fait l'objet de nombreuses études et interprétations. En 2002 les Rencontres Nationales de Patristique de Carcassonne ont retenu pour thème «le culte des reliques au $\mathrm{IV}^{\mathrm{e}}$ siècle» et les actes viennent d'être publiés dans un numéro complet de Connaissance des Pères de l'Eglise fort intéressant $\left({ }^{3}\right)$. À partir de la Bible, Paul-Irénée Fransen montre les premiers développements du culte des reliques, partie intégrante de la piété chrétienne qui «exprime sans doute le meilleur de l'homme». Le culte des martyrs est évoqué par François Heims à partir des études anciennes et classiques du bollandiste Hippolyte Delehaye (1933) et d'Ernest Lucius (1904), et de celles récentes de Monseigneur Victor Saxer et de Noël Duval. Pierre Maraval, spécialiste des lieux saints, retrace les grandes étapes de la découverte de la Croix au IV $\mathrm{V}^{\mathrm{e}}$ siècle.

Le thème difficile, car fécond et ardu, de la «théologie des reliques» est abordé par Paul Force à partir de l'exemple de Vigilance de Calagurris, prêtre du Comminge à la fin du IV ${ }^{e}$ siècle, et de sa critique du culte des reliques, en particulier à propos de saint Saturnin à Toulouse. Pour Vigilance «le culte adressé aux martyrs est emprunté aux manifestations cultuelles de la Rome païenne et est entaché d'idolâtrie. Les baisers apposés sur les reliques, les processions de reliques dans les églises et dans les rues, les prosternations devant les reliques, l'utilisation de linges précieux pour envelopper des

(1) Le premier article a paru ici-même, t. LXXX, 2002, p. 563-591. Devra-t-on un jour rédiger une bibliographie de l'histoire des reliques ou mieux la mettre sérieusement et systématiquement sur Internet ? Comme le sujet se prête également idéalement à l'interdisciplinarité, il élargit ipso facto la sphère de recherches et son ouverture aux expositions accroît davantage encore son actualité .

(2) Cambridge, University Press, 2001, p. 283-318.

(3) $\mathrm{N}^{\circ} 89,2003$. 
cendres, ce sont là des rites païens. Et le sacrilège est d'autant plus patent que ces rites sont pratiqués au cours de la liturgie chrétienne: on est donc en présence d'une modification du rituel de la liturgie. Nous voyons donc ici le double plan sur lequel se place Vigilance: refus d'attitudes païennes, et refus de mettre la liturgie des synaxes au service de ces manifestations païennes. Il refuse aussi qu'on associe le corps du martyr et l'Eucharistie dans une réunion ecclésiale. Mais Vigilance se trouve à contre-courant de Jérôme ou d'Augustin. C'est un moment-clé de l'histoire des reliques: saint Martin meurt vers 397, comme saint Ambroise de Milan, qui donne des reliques des saints Nazaire, Protais et Gervais à saint Victrice de Rouen qui immortalise leur arrivée à Rouen dans son De laude sanctorum (397). Ce récit, calqué sur celui de l'entrée de l'empereur dans la cité (adventus), fera florès dans le sud de la Gaule; Paulin de Nole vante Victrice. C'est le début des translations de reliques. C'est l'époque où le pape Damase organise le culte des martyrs dans les catacombes et compose, puis fait graver les fameuses épitaphes. Le traité de Jérôme développe toute cette nouvelle théologie des reliques dans un syncrétisme religieux prudent. Plus tard Augustin prolongera ces réflexions et, en 421 dans son De cura pro mortuis gerenda, il admettra la protection des morts par les saints. L'attitude d'Augustin envers les reliques s'est en effet modifiée au cours de sa vie, sans doute précisément à la suite du développement du culte des martyrs à son époque: Gervais, Protais, mais surtout en 424 l'arrivée des reliques d'Étienne à Hippone. Le culte des reliques dans la Cité de Dieu (Livre X) conduit au Christ ressuscité. Ce sens théologique qu'Augustin définit est étayé par les miracles suscités par les reliques dans sa vision d'une foi en la Résurrection: à la différence du paganisme, le christianisme n'est pas une religion de morts mais de vivants. Augustin insiste bien sur la différence entre le culte rendu à Dieu (adoration) et la vénération des reliques. "À cette nouvelle vision de l'homme qu'elles induisent s'ajoute une vision renouvelée de l'Eglise, un rapport plus fort aux confesseurs de la foi. Cela se manifeste par les autels construits sur les tombeaux des martyrs, qui justement, contiennent des reliques. Le caractère fondateur des reliques est ainsi mis en évidence. Le lien entre les reliques et l'Eucharistie apparaît aussi dans la mesure où les reliques amènent à mieux comprendre le sens de l'Eucharistie, le mémorial qui est célébré, le sacrement de la Résurrection qui est donné. En raison de sa densité anthropologique et ecclésiale, on comprend que le culte des reliques qui s'est développé dans les premiers siècles, nous parle encore aujourd'hui, en particulier en raison de l'humanité glorifiée, de la vision de l'être humain en relation avec le Christ qu'ils proposent et de la présentation du christianisme comme religion de vivants» (Marie-Anne Vannier).

Le numéro spécial de Connaissances des Pères de l'Eglise aborde d'autres thèmes dont celui du rapport «reliques-villes» : tumulatio ad sanctum, suburbium martyrial. Lucie Piétri l'avait bien montré avec l'exemple de Tours $\left({ }^{4}\right)$. Le développement de Narbonne est analysé par André Bonnery. La

(4) L. PIÉTRI, La ville de Tours du IVe au VIe siècle. Naissance d'une cité chrétienne, École Française de Rome, 1983. 
tombe vénérée de Paul entraîne la construction de plusieurs églises successives auxquelles fut adjoint un monastère et l'urbanisation du suburbium sur la rive droite de l'Aude se poursuit : le burgus sancti Pauli devient aussi important que celui de la vieille cité antique de la rive gauche.

\section{Poursuivre l'inventaire des trésors}

Comme pour toute collection muséale, seul un inventaire scientifique exhaustif des trésors de reliques et leur édition critique contribueront à mieux les faire connaître.

Extraordinaire, le mot n'est pas trop fort pour qualifier l'inventaire des reliques de Cologne par Hans-Joachim Kracht et feu Jacob Torsy $\left({ }^{5}\right)$. Tout qui s'est quelque peu frotté à l'édition de sources concernant un trésor d'église, quel qu'il soit, mesure toute l'ampleur du travail lorsqu'on passe à l'échelle colonaise : "Gaude, felix Agrippina sanctaque Colonia» de tant de saints, donc de tant de reliques! En 1910 Georges Zilliken avait donné une étude magistrale pour l'époque du calendrier colonais. La voici aujourd'hui complétée d'une recherche sur les reliques des saints conservées dans les églises de Cologne $\left({ }^{6}\right)$. Le tableau hagiographique colonais s'achève. Un pareil travail n'arrive pas tout seul. Des études préparatoires ont été nécessaires : on pense en particulier aux nombreuses recherches d'Anton Legner et à ses expositions Ornamenta ecclesiae de 1985. Jacob Torsy n'est pas en reste avec ses synthèses dans les catalogues Rhin-Meuse (1972) et Monumenta Annonis (1985) mais aussi ses nombreux livres et articles sur les saints régionaux et sur les dédicaces. L'archevêque de Cologne Annon (†1075), à lui seul, est un de ces «fous» de reliques et... finit lui-même comme relique et insigne cadeau offert à sa fondation chérie de Siegburg par sa canonisation vers $1183-1186$.

Marie-Madeleine Gauthier insistait - nous l'avons plus d'une fois rappelé - sur cette publication systématique des trésors d'églises $\left({ }^{7}\right)$. Publica-

(5) H.-J. KRACHT et J. TORSY ( $\dagger$ ), Reliquiarium Coloniense, Siegburg, 2003 (Studien zur Kölner Kirchengeschichte, t. XXXIV).

(6) Les saints sont énumérés par ordre alphabétique de la p. 77 à la p. 521, situés par leurs coordonnées hagiographiques et par une courte biographie avec bibliographie; ensuite inventaire est fait d'après les sources de la présence de leurs reliques à Cologne. Les patronymes et dédicaces d'autels sont ègalement étudiés. Des lettres en gras indiquent directement si les saints sont mentionnés dans le trésor (A), dans la consécration d'autels (B), dans le titre d'autels ou de chapelles (C), dans le nom d'une confrérie (D) ou de cloches (E). La sphère des reliques est ainsi même parfois élargie : le titre d'un autel n'implique pas obligatoirement la présence de reliques du saint invoqué dans sa table.

(7) Quelques exemples récents à retenir : M. L. DE KREEK, De Kerkschat van het OnzeLieve-Vrouwekapittel te Maastricht, Utrecht, 1994 (Clavis Kunsthistorische Monografieën, t. XIV) ; U. GROTE, Der Schatz von St. Viktor. Mittelalterliche Kostbarkeiten aus dem Xantener Dom, Ratisbonnc, 1998 ; L. BECKS et R. LAUER, Die Schatzkammer des Kölner Domes, Cologne, 2000 et T. HUSBAND et J. CHAPUIS, The Treasury of Basel Cathedral, New York, The Metropolitan Museum of Art, 2001. 
tion et interprétation doivent être associées. L'abbaye bénédictine de Zwiefalten près d'Ulm en Souabe, dont les sources écrites concernant les reliques (dédicaces d'autels, inventaire, obituaire) sont bien éditées, eut des relations privilégiées avec l'Est, la Pologne en particulier. Edina Bozoky s'intéresse à la translation de la relique de la main de saint Étienne, récit de 1140 dû à un moine de Zwiefalten $\left({ }^{8}\right)$, dont elle donne une traduction française. Le bras droit du saint avait une valeur particulière, étroitement liée au pouvoir impérial de Constantinople. "Les bras et les mains figuraient par ailleurs parmi les plus précieuses parties des corps saints après la tête, puisque c'est avec leur bras et leur main que, de leur vivant, les saints accomplissaient les gestes de bénédiction et de guérison, et transmettaient ainsi la virtus divine». Au début du XII ${ }^{\mathrm{e}}$ siècle Barbara Comnène, princesse porphyrogénète, épousa Sviatopolk Michaël, prince de Kiev. Le prince byzantin dota sa fille de divers trésors et... de reliques dont cette main droite du protomartyr Étienne. Le récit raconte l'acquisition de la précieuse relique par le duc de Pologne Boleslaw III et son don par la veuve du duc après 1138 à l'abbaye. En une cinquantaine d'années la relique passe de Constantinople à Zwiefalten, en faisant étape à $\mathrm{Kiev}$ et en plusieurs localités polonaises. La circulation des biens entre l'Est et l'Ouest en est bien mise en évidence. La circulation des reliques par dot et par douaire, vraisemblablement très répandue au Moyen Age, est mentionnée dans quelques textes hagiographiques $\left({ }^{9}\right)$.

Le pays mosan développe lui-aussi de nombreux contacts reflétés par l'histoire des reliques. L'abbaye du Neufmoustier près de Huy a ainsi rassemblé une collection de reliques de Terre Sainte qui conduit sur les routes de pèlerinage $\left({ }^{10}\right)$. En 2004 le Musée diocésain de Mayence fut à l'heure des croisades. L'exposition mit en scène dans une chapelle-trésor suggérée quelques reliquaires intéressants: le verre de sainte Hedwige, le bonnet de saint Bernard, la croix de Brogne, la croix-reliquaire de Scheyern, la staurothèque de Denkendorf, ... ou le doigt de saint Jacques d'Eichstätt. Une partie importante du livre-catalogue est consacrée à Die Macht der Reliquien : Kreuzfahrer zwischen religiösen Motivation und Gewinnsucht, avec l'information générale utile Zum Wesen der Reliquien, Herrenreliquien, Reliquienhandel, Reliquienraub, illustrée notamment par les détails de la châsse limousine de saint Saturnin de Toulouse (1176-1198) présente à l'exposition.

(8) Ed. BOZOKY, "Le Trésor de reliques de l'abbaye de Zwiefalten. Un précieux témoignage des échanges culturels entre Orient et Occident", dans Les échanges culturels au Moyen Age. XXXIle Congrès de la Société des Historiens Médiévistes de l'Enseignement Supérieur Public, Université du Littoral Côte d'Opale, juin 2001, Paris, 2002, p. 117-133. Cet ouvrage s'intéresse plus largement au système de l'échange de type symbolique qui est au cour de toute vie sociale au Moyen Age.

(9) Ed. BOZOKY, "Le rôle des reines et princesses dans les translations de reliques", dans C. RAYNAUD, éd., Reines et princesses au Moyen Age. Actes du cinquième colloque international de Montpellier, Université Paul-Valéry, 24-27 novembre 1999, Montpellier, 2001 (Les Cahiers du CRISIMA., $\mathrm{n}^{\circ}$ 5) , p. 349-360.

(10) $\mathrm{Ph}$. GEORGE, "Le trésor de reliques du Neufmoustier près de Huy. Une part de Terre sainte en pays mosan", dans Bulletin de la Commission Royale d'Histoire, t. CLXIX, 2003, p. 17-35. 


\section{Développer la grille de lecture}

D'étude en étude, de nouveau thème en nouveau thème, la grille de lecture peut donner des idées et être ainsi déplacée géographiquement; en même temps de région en région la problématique s'enrichit.

Le plus neuf pour la recherche scientifique est l'analyse minutieuse des échanges, des contacts mentionnés dans les sources historiques et corroborés par les reliques retrouvées et les reliquaires conservés. Beaucoup de personnalités en sont exemplatives. A cet égard les confraternités se sont avérées très utiles. Cette union spirituelle de deux établissements religieux dans la prière commune et réciproque pour les vivants et les morts permet aussi une entraide matérielle dont les modalités varient. Les confraternités apportent un témoignage concret des liens qui unissent deux établissements religieux et nous avons pu constater, à partir du cas de Stavelot-Malmedy, que les transferts de reliques ont suivi le même chemin, suscités ou favorisés par ces associations, parfois de manière fortuite. Tout ceci s'inscrit largement dans cette anthropologie médiévale chère à Jacques Le Goff et à Jean-Claude Schmitt.

Les reliques de La Voûte-Chilhac en Auvergne sont connues par un inventaire du $\mathrm{XI}^{\mathrm{e}}$ siècle exploité par Edina Bozoky $\left({ }^{11}\right)$. La relique insigne est un fragment du Bois de la Vraie Croix offert par l'impératrice Cunégonde, l'épouse d'Henri II, à replacer dans la dévotion particulière de l'empereur et de Cluny pour le Saint Bois. Une collection de reliques (90 reliques), rassemblées en un laps de temps assez court, par «lots», la plupart obtenues de l'abbaye de Saint-Denis par les liens privilégiés d'Odilon de Cluny avec ce monastère. L'emplacement des reliques dans l'église est difficile à préciser. A titre d'exemple, le Liber Tramitis de Cluny, rédigé à l'époque d'Odilon, nous renseigne précisément sur l'utilisation liturgique des reliques. C'est le dimanche des Rameaux que toutes les reliques sont transportées en procession. La veille de la fête, on disposait les châsses derrière le maître-autel sur des brancards en bois. La procession avec les reliques avait lieu après la bénédiction des palmes et d'autres branches. L'ordre du cortège était rigoureusement fixé : les convers portaient quatre croix, deux encensoirs d'or, de l'eau bénite, quatre cierges; un prêtre prenait le bras de saint Maur; la statuereliquaire de saint Pierre, le corps de saint Marcel et la châsse du pape Grégoire, et enfin les reliques de beaucoup d'autres saints étaient portées respectivement par deux ou quatre frères convers. De même, lors des Rogations, les reliques occupaient une place importante dans la procession et les litanies. Ce qui amène l'auteur à s'interroger plus largement sur la fonction et la signification des collections de reliques. «La collection de reliques de $\mathrm{La}$ Voûte est parfaitement représentative pour la première moitié du $\mathrm{XI}^{\mathrm{e}}$ siècle. C'est la période où l'utilisation politique et religieuse des rassemblements de reliques atteint probablement son apogée. Moyens pour garantir la paix des âmes de la parentèle d'Odilon, ces reliques reflètent les liens d'Odilon - et de

(11) Ed. BOZOKY, "Les reliques de La Voûte", dans Odilon de Mercoeur. L'Auvergne et Cluny. La "Paix de Dieu» et l'Europe de l'an mil, Actes du Colloque de Lavoûte-Chilhac, 10-12 mai 2000, Nonette, 2002, p. 175-190. 
Cluny - tant avec les pouvoirs politiques qu'avec toute la hiérarchie des puissances célestes présentes à La Voûte».

Le 13 novembre 1004, à La Réole sur les bords de la Garonne, fut tué l'abbé Abbon de Fleury, victime d'une rixe entre gascons et fleurisiens. En 2004 Saint-Benoît-sur-Loire célébra le millénaire du saint par un colloque $\left({ }^{12}\right)$ et par une exposition $\left({ }^{13}\right)$. En outre Pierre Riché a consacré une belle biographie à cet écôlatre illustre, contemporain de Gerbert $\left({ }^{14}\right)$. Depuis 653 au plus tard Fleury s'enorgueillit de conserver les précieuses reliques de saint Benoît. Les textes et les fouilles archéologiques ont permis à Sébastien Jesset, dans le très remarquable catalogue, de tirer plusieurs conclusions sur lc sćpulcre de saint Benoît au haut Moyen Age : "Les reliques de saint Benoît furent d'abord conservées dans une urna, déposées en-dessous du niveau du sol de la basilique, vraisemblablement dans une fosse aménagée et dont l'emplacement était marqué au sol. Au-dessus de cette fosse, peut-être close par une dalle, devait s'élever un monument en pierre, lourd et assez haut, pouvant consister en un autel-reliquaire». Après leur fuite devant les Normands et l'incendie de l'abbaye en 865 , les moines reconstruisirent à la croisée du transept une structure pour accueillir les reliques et les mettre en évidence par un dallage circulaire de pierres en opus sectile surmontant seize vases enfouis dans le sol et peut-être destinés à les préserver de l'humidité. Le comblement et l'abandon de ce sépulcre intervint après 1026 et avant 1030.

L'exposition rassembla des œuvres de toute première importance. Danièle Gaborit-Chopin signe le chapitre sur les arts précieux à Fleury et Jean-Pierre Caillet une recherche sur le reliquaire de Mumma (VII ${ }^{\mathrm{e}}$ siècle?), découvert en 1642 sous le maître-autel de l'abbatiale : Mumma fieri iussit $i(n)$ amore $s(a n) c(t)$ e Marie et $s /(a n) c(t) i$ Petri : Mumma est-il l'abbé Mummolus qui fit agrandir l'un des deux sanctuaires de Saint-Benoît-sur-Loire pour y transférer les reliques de saint Benoît? Selon Éliane Vergnolle au colloque, la chapelle Saint-Mommole, petit bâtiment rectangulaire qui, au niveau de la crypte, flanque au sud le choeur de l'abbatiale, aurait été la salle du trésor, le gazophylacium d'Abbon.

D'un premier colloque international à Boulogne dont elle fut l'une des chevilles ouvrières, à une habilitation à Poitiers intitulée Reliques, pouvoir et société au Moyen Age. La politique des reliques (IVe-XIIIe siècle). De la protection collective à la légitimation du pouvoir $\left({ }^{15}\right)$, Edina Bozoky multiplie avec perspicacité et brio les études sur les reliques. Après l'exemple de la

(12) Abbon de Fleury, un abbé de l'an mil. Colloque international organisé par l'Institut de Recherche et d'Histoire des Textes et l'Abbaye de Saint-Benoît-sur-Loire (10-12 juin 2004).

(13) Lumières de l'an mil en Orléanais. Autour du millénaire d'Abbon de Fleury, Orléans Musée des Beaux-Arts, Turnhout, Brepols, 2004, rassemble près d'une cinquantaine d'éminents collaborateurs dans des chapitres sur Carolingiens et Capétiens en Orléanais, Orléans aux $X^{e}-X I^{e}$ siècles, Campagnes, bourgs et châteaux, Les grands monastères, Art, science et savoir, avec tous les index nécessaires, $279 \mathrm{p}$.

(14) P. RICHÉ, Abbon de Fleury, Turnhout, Brepols, 2004.

(15) Poitiers, Centre d'Etudes Supérieures de Civilisation Médiévale, 15 décembre 2003. 
Flandre sous ses premiers comtes aux $\mathrm{IX}^{\mathrm{e}}-\mathrm{XI}^{\mathrm{e}}$ siècles, elle pousse plus loin l'enquête sur l'initiative et la participation du pouvoir laïque dans les translations de reliques au haut Moyen Age et en esquisse une typologie à l'échelle européenne $\left({ }^{16}\right)$. Ces translations s'inscrivent en effet dans une construction de la topographie sacrée urbaine et constituent des indicateurs précieux sur les enjeux du pouvoir dans la société médiévale. Leur préfiguration trouve origine dans la Bible - l'Arche d'alliance - ainsi que dans les adventus, les «joyeuses entrées», des empereurs romains. Ce n'est pas un hasard si Arnold Angenendt a choisi pour la couverture de son livre sur les reliques la plaquette d'ivoire du Trésor de Trèves qui montre le cortège rituel de reliques dans une ville vers $500\left({ }^{17}\right)$. Le parallélisme établi par Edina Bozoky avec le pouvoir impérial romain peut être prolongé sur le plan liturgique en évoquant la récupération de diptyques consulaires sur l'autel chrétien pour servir de support au texte du memento de la messe. Leur souvenir à Tongres ou à Liège inclut des saints évêques. Constantinople, à ses origines ville pauvre en reliques, s'en enrichira progressivement par des translations d'initiative publique. En Occident les Pippinides inaugurent cette politique de reliques. L'affirmation du pouvoir royal, plus tard seigneurial, s'y détecte, même si le peuple ou le clergé - les évêques - sont à la base de la majorité des translations. Le pouvoir séculier aspire au contrôle des reliques, enjeu de pouvoir, donc de prestige et de gloire.

En marge de 1'exposition de Saint-Riquier $\left({ }^{18}\right)$, un colloque fut organisé par l'Université de Picardie. Edina Bozoky y étudie le recouvrement des reliques des saints Valéry et Riquier par Hugues Capet en 981, dans le cadre d'une légitimation du pouvoir capétien et de la diffusion des réformes monastiques $\left({ }^{19}\right)$. Ce recouvrement connut un développement légendaire : le poème d'Angelramne, abbé de Saint-Riquier (1022-1045), consacré au retour des reliques de saint Riquier, présente de manière flatteuse Hugues Capet, descendu de cheval, nu-pieds, portant le reliquaire sur ses épaules jusqu'au sanctuaire. On se souviendra que, conjointement au rétablissement d'une vie monastique régulière, le réformateur Gérard de Brogne $(\dagger 959)$ visait à la reconstitution du temporel des établissements réformés. Ici les reliques légitiment les prétentions monastiques (Fontenelle/Saint-Wandrille) mais aussi,

(16) Ed. BOZOKY, "L'initiative et la participation du pouvoir laïc dans les translations de reliques au haut Moyen Age. Esquisse typologique", dans Sources. Travaux historiques, $\mathrm{n}^{\circ}$ 51-52, 1997, p. 41-57. On relèvera également du même auteur "Le culte des saints et des reliques dans la politique d'Henri II et de Richard Cour de Lion", dans M. AURELL, ed., La cour Plantagenêt (1154-1204). Actes du colloque international, Thouars 1999, Poitiers, Centre d'Etudes Supérieures de Civilisation Médiévale, 2000 (Coll. Civilisation médiévale, t. VIII), p. 277-291.

(17) A. ANGENENDT, Heilige und Reliquien, Munich, 1994, et notre compte-rendu dans Francia. t. XXIV, 1997, p. 170-171.

(18) Cf. notre premier article cité en n. 1, p. 580.

(19) Ed. BOZOKY, "Le recouvrement des reliques des saints Valéry et Riquier par Hugues Capet", dans D. BUSCHINGER, ed., Saint Riquier à Saint-Riquier. Actes du Colloque du Centre d'Etudes Médiévales de l'Université de Picardie Jules Verne, Saint-Riquier 9 et 10 décembre 2000, Amiens, 2001 (Médiévales, t. XIII), p. 1-13. 
comme en Flandre, Hugues Capet, promoteur du mouvement de réforme, s'en sert à son profit. "La récupération des reliques avait une fonction spécifique dans ce processus: tout d'abord, pour la consécration des autels ainsi que pour la prononciation des vœux des moines, il était nécessaire de posséder des reliques; mais aussi, la présence de reliques importantes, qui produisaient des miracles, attirait des donations et des aumônes, et contribuait ainsi au renouveau matériel des monastères ". Le miracle intervient et sublime la virtus des reliques. Les hagiographes montrent tout l'effet bénéfique des reliques. Sous leur plume le rêve et la prophétie deviennent un instrument de légitimation du pouvoir. Qui se sert de qui? Le religieux du laïc et vice versa. La question ne se pose pas au Moyen Age. Légitimité du pouvoir royal capétien et transfert des carolingiens aux capétiens, rapatriement des reliques importantes pour le développement du monastère. La problématique est ancienne : on pense tout naturellement aux rois thaumaturges de Marc Bloch. La trésorerie de Saint-Riquier possédait un doigt de saint Marcoul. Des peintures gothiques tardives semblent rappeler les liens entre le pouvoir royal et celui des saints. Pour mémoire à Malmedy, au XVI $\mathrm{X}^{\mathrm{e}}$ siècle, les Miracles de saint Quirin mentionnent aussi saint Marcoul $\left(\mathrm{VI}^{\mathrm{e}}\right.$ siècle) dont les reliques furent habilement exploitées en un pèlerinage renommé à Corbény (France, Aisne), prieuré dépendant de l'abbaye de Saint-Remi de Reims; les bénédictins rémois entretinrent la réputation de spécialisation thaumaturgique du saint pour guérir des écrouelles, en liaison directe avec le pouvoir reconnu à la royauté française. Dans le Miracle en question, l'échec d'un premier pèlerinage à saint Marcoul peut accréditer un pouvoir supérieur reconnu à saint Quirin, patron de Malmedy $\left({ }^{20}\right)$.

Les symboles du pouvoir sont concernés : sainte Ampoule, oriflamme de saint Denis, Henri ${ }^{\text {er }}$ et la sainte Lance... Les reliques gagnent une présence qui renforce leur rôle dans la représentation du soutien surnaturel du pouvoir terrestre.

Entre le $X^{e}$ et le $X I^{e}$ siècle, en Gaule du Nord et de l'Ouest ainsi qu'en Angleterre, une littérature diffuse le scénario hagiographique-type de découverte par le petit peuple de l'emplacement de reliques connues et oubliées. Ces récits encouragent la réforme d'établissements aux $\mathrm{X}^{\mathrm{e}}-\mathrm{XI}^{\mathrm{e}}$ siècles qui avaient besoin de reliques pour rehausser leur prestige. L'intervention du peuple sert à légitimer de nouveaux cultes suivant l'adage bien connu Vox populi, vox Dei. La solidarité de la société s'exprime autour du nouveau saint $\left({ }^{21}\right)$.

Parmi les topoi hagiographiques, la lumière accompagne les reliques des saints par des phénomènes les plus divers $\left({ }^{22}\right)$. Sa fonction est importante: la

(20) Ph. GeORge, "Les Miracles de saint Quirin de Malmedy, un livret médiéval au coeur du XVI ${ }^{\mathrm{e}}$ siècle", dans Bulletin de la Commission Royale d'Histoire, t. CLXIV, 1998, p. 1-29.

(21) Ed. BOZOKY, "Le rôle du petit peuple dans les inventions de reliques", dans $P$. BOGLIONI, R. DELORT \& Cl. GAUVARD, eds., Le petit peuple dans l'Occident médiéval. Terminologies, perceptions, réalités. Actes du Congrès international de Montréal, Paris, Publications de la Sorbonne, 2002, p. 549-558.

(22) Ed. BOZOKY, "La lumière des reliques", dans Pris-MA. Recherches sur la littérature d'imagination du Moyen Age, t. XVIII, $\mathrm{n}^{\circ}$ 35-36, 2002, p. 16-30. 
lumière sublime les reliques; elle peut aussi révéler l'emplacement sacré pour l'inventio. Ici le Moyen Age a la vie longue : au XVII ${ }^{\mathrm{e}}$ siècle par exemple la révélation du corps d'un saint martyr sarde à un pèlerin liégeois s'accomplit encore de la même manière; il découvre un sépulcre et en avertit l'Archevêque de Cagliari lequelle me defendit du toucher iusque a ce qu'il seroit present. Icelluy arrivé me commandat de descendre dans le dict sepulchre, et voulant eslever la pierre qu'estoit aux pieds, appareurent de la lumiere dans le dict sepulchre, tout estonné ie la laissast retomber, ce que voyant l'Archevesque m'at demandé ce qu'il y avoit; auquel respondit que iavoit veu de la lumiere et mexhortant de prendre couraige, me dist que s'estoit choese ordinaire anciennement de mettre de la lumiere dans les sepulchres, et puis $m$ 'ayant commandé de luy donner la lampe, et d'ouvrir tout le sepulchre. Ce qu'ayant faict, et icelluy voyant le corps si beau me fit sortir, et y fist descendre quattre Prestres pour l'eslever [...] $\left({ }^{23}\right)$. Et des exemples existent jusqu'au XIX ${ }^{\mathrm{e}}$ siècle...Décidément le halo de lumière entourant le saint impressionne de tous temps.

La réimpression d'articles, entreprise toujours si utile au vu de leur dispersion, se signale à notre attention: Jacques Paul à propos de miracles du sud de la France $\left({ }^{24}\right)$, ou Henri Platelle $\left({ }^{25}\right)$ sur Guibert de Nogent et son De pignoribus sanctorum mais aussi sur des thèmes hagiographiques comme "la mort précieuse» ou «le cadavre qui saigne en présence de son meurtrier»; et, dans un registre semblable, les Mélanges Michel Parisse ont permis d'offrir au Maître quelques articles reliquiophiliques: Dominique Iogna-Prat et le rituel de dédicace, Eric Palazzo et les arts somptuaires.... ou Guy Philippart à propos d'hagiographie namuroise $\left({ }^{26}\right)$.

\section{L'interdisciplinarité plus que jamais d'actualité}

C'est peut-être la force de la «reliquiologie» et plus largement de l'hagiologie que de brasser avec bonheur de nombreuses disciplines.

Dans une remarquable exposition internationale itinérante, Didier Méhu aborde les reliques sur «les chemins du Moyen Âge» $\left({ }^{27}\right)$, illustrant son propos sur «les saints protecteurs» par quelques belles châsses limousines.

(23) Ph. GEORGE, "Revenant et exorcisme à Liège. Quête de reliques en Sardaigne (1634-1652)", dans Bulletin de la Commission Royale d'Histoire, t. CLXVII, 2001, p. 253305.

(24) J. PAUL, Du monde et des hommes. Essais sur la perception médiévale, Aix-enProvence, Publications de l'Université, 2003.

(25) H. Platelle, Présence de l'au-delà. Une vision médiévale du monde, Lille, Presses universitaires, 2004.

(26) Retour aux sources. Textes, études et documents d'histoire médiévale offerts à Michel Parisse, Paris, Picard, 2004.

(27) D. MEHU, Gratia Dei. Les chemins du Moyen Age, Québec, Musée de la Civilisation, 2003, 224 p. et traduction anglaise (Grand-Rapids, 2004) et allemande (Münster et Liège, 2004-2005), p. 109 sv.. 
Les recueils de miracles des $\mathrm{V}^{\mathrm{e}}-\mathrm{VII} \mathrm{I}^{\mathrm{e}}$ siècles permettent à Edina Bozoky une perception de l'édifice cultuel dans l'imaginaire. Le rapport qui existe entre le saint, son sanctuaire et son activité thaumaturgique met en relief avant tout la présence vivante du saint. «Dans les recueils de miracles orientaux (Thècle, Côme et Damien, Cyr et Jean, Artémios), le sanctuaire apparaît comme une demeure comparable à une maison profane, où le saint agit comme les êtres vivants, ayant ses habitudes, voire ses soucis. Les apparitions de saints sont qualifiées souvent de "vision réelle». La "corporéité» des saints et leur ancrage à leur sanctuaire sont soulignés même dans les miracles relatant les sorties des saints. En Occident, la présence vivante des saints caractérise également les livres des miracles de Grégoire de Tours, mais l'aspect profane de l'église "habitée» par les saints disparaît; désormais l'accent est mis sur leurs reliques et sur le caractère proprement cultuel de leur sanctuaire $»\left({ }^{28}\right)$.

Qu'il soit réel ou imaginaire, l'espace sacré a retenu l'attention du Centre d'Etudes Médiévales d'Auxerre. En juin 2003 l'Atelier sous la responsabilité d'Edina Bozoky eut pour but d'approfondir un aspect essentiel de ce thème : le lien entre reliques et espace comme forme de pratiques sociales (IX ${ }^{\mathrm{e}}-\mathrm{XII}{ }^{\mathrm{e}}$ siècles) $\left.{ }^{29}\right)$.

Fondées essentiellement sur des sources textuelles - hagiographiques, historiographiques mais aussi théologiques - les contributions ont analysé l'utilisation des reliques pour marquer l'espace sacré, ecclésiastique et politique, ainsi que pour exprimer les identités communautaires également inscrites dans l'espace. La distribution spatiale des reliques, lors de leur déposition dans une église, ne reflète pas simplement la hiérarchie des saints établie selon l'usage liturgique. L'importance et l'origine des acquisitions, le choix effectué dans leur mise en valeur peuvent indiquer également la place d'une église dans le réseau des sanctuaires à l'échelle d'une région, d'une circonscription ecclésiastique ou d'un pays. En même temps, les initiatives de récupération et de collecte de reliques sont intimement liées à la réforme monastique et ecclésiastique des $\mathrm{XI}^{\mathrm{e}}-\mathrm{XI}^{\mathrm{e}}$ siècles (Anne-Marie Helvétius, Benoît Tock, Anne Wagner). Anne Wagner a terminé l'édition des trésors de Verdun. Dans une autre perspective, les exodes et les recouvrements des reliques aux frontières de la Normandie témoignent aussi des problèmes de la territorialité du pouvoir (Jacques Le Maho). La correspondance entre reliques et Eucharistie concerne non seulement leurs fonctions cultuelles, mais joue également un rôle déterminant dans la formation et les développements d'une doctrine du lieu de culte (Dominique Iogna-Prat). Une analyse du traité de Thiofrid, abbé d'Echternach (Flores epytaphii sanctorum, fin $\mathrm{XI}^{\mathrm{e}}$-début $\mathrm{XII}^{\mathrm{e}}$ siècle) permet de voir l'articulation entre la mise en scène du Christ dans l'Eucharistie et celle des saints dans leurs reliques (Frédéric Munier). En dehors des

(28) Ed. BOZOKY, "La «maison» du saint et les miracles", dans Hortus artium medievalium. Journal of the International Research Center for the Late Antiquity and Middle Ages, t. IX, Zagreb-Motovun, 2003, p. 247-254.

(29) Cf. Etudes et travaux 2003-2004, Auxerre, Centre d'Etudes Médiévales, 2004, p. 57-59. 
translations cérémonielles de reliques, leur circulation selon d'autres modalités est partie constituante d'un système d'échanges plus large centré sur le don au cœur des relations entre ici-bas et au-delà (Eliana Magnani). La littérature hagiographique révèle plus ou moins explicitement les raisons profondes des translations de reliques. La politique épiscopale du culte des saints à Naples peut être interprétée à la lumière des récits de transferts composés aux $\mathrm{IX}^{\mathrm{e}}$ et $\mathrm{X}^{\mathrm{c}}$ siècles (Thomas Granier). L'examen du vocabulaire et des discours portant sur le lien entre l'acquisition de reliques et la protection du royaume montre comment ce thème devient progressivement une expression de l'idéologie du pouvoir royal (Edina Bozoky). Pour notre part nous y avons évoqué les reliques en pays mosan en marge de notre édition des trésors, en faisant le lien entre la sacralisation de l'espace et la thaumaturgie spécialisée.

La Dedicatio Stabulensis est un fort intéressant texte qui relate la consécration de la grande abbatiale bénédictine de Stavelot le 5 juin 1040 en présence de la cour royale $\left({ }^{30}\right)$. Deux ans plus tard la redécouverte des lieux de culte et des reliques de Remacle, le saint patron fondateur, stimule le pèlerinage au saint ardennais (Inventio Stabulensis). Les deux récits contemporains des événements, Dedicatio et Inventio, s'inscrivent plus largement dans un désir des réformateurs de magnifier leurs nouvelles constructions comme la figure idéale de l'Eglise chrétienne, à une époque troublée par des bruissements d'hérésie. Le corpus de dédicaces d'églises autour de l'an mil est assez restreint $\left({ }^{31}\right)$. Dominique Iogna-Prat considère la Dedicatio comme 1'un des premiers témoins chronologiques d'un type historiographique nouveau, le $D e$ constructione-de consecratione ecclesie, genre de panégyrique de monument, dont l'exemple le plus achevé sera le célèbre traité De consecratione de l'abbé Suger (1081-1151). Aux simples notices assez sèches de dédicaces, dont les exemples dans l'Empire sont nombreux, succède une vraie «célébration monumentale monastique " : les réformateurs monastiques contribuent à une exceptionnelle floraison monumentale. Poppon de Stavelot suit l'exemple de son maître Richard de Saint-Vanne qui, dans l'au-delà, à en croire Pierre Damien, continuait pour sa peine à élever de vaines constructions de pierre.

L'histoire de l'art se décline aussi par les reliques. Qui dit «reliques» dit «édifices» pour les abriter. Jean-Pierre Caillet s'est livré à un panorama des réalisations les plus remarquables qu'a pu connaître Sylvestre II, qui ne semble pas avoir été lui-même un grand bâtisseur $\left({ }^{32}\right)$. Les bâtisseurs de l'époque s'efforcent «de répondre au mieux à des exigences liturgiques et dévotionnelles précises : protection et mise en valeur des reliques dont on se trouvait en possession de plus ou moins longue date; en relation avec ces

(30) Ph. GeOrge, “Les reliques de Stavelot et de Malmedy à l'honneur vers 1040. Dedicatio et Inventio Stabulensis", dans Revue d'Histoire Ecclésiastique, 2004, p. 347-370.

(31) D. IOGNA-PRAT, "Les moines et la «blanche robe d'églises" à l'âge roman", dans Actes des Journées romanes d'Estella (2003), Pamplona, 2004, p. 319-347, fait l'inventaire et un excellent commentaire des dédicaces d'églises.

(32) J.-P. CAILlET, "Les horizons monumentaux de Gcrbert d'Aurillac", dans F1. G. NUVOlone, ed., Gerberto d'Aurillac da Abate di Bobbio a Papa dell'Anno 1000. Actes du Congrès international de Bobbio (2000), Archivum Bobiense Studia, t. IV, 2002, p. 681-698. 
reliques, multiplication des pôles cultuels pour permettre à chaque moineprêtre de célébrer quotidiennement sa messe; facilité d'accès à ces pôles pour les religieux eux-mêmes et éventuellement, déjà, pour les fidèles». Il examine ainsi successivement l'Auvergne, la Catalogne, Reims, Orléans, Ravenne et Rome. L'an mil en histoire de l'art en France ne peut faire l'économie de la fameuse citation de Raoul Glaber qui, d'un point de vue lotharingien et plus encore germanique, paraît décalée quant à la réalité historique. L'expression «autour de l'an mil» n'en acquiert que plus de pertinence: 970-980 à 10301040 s'érige en phase riche en constructions majeures. Tout est question d'appréciation entre «les premiers frémissements», la préfiguration et la pleine affirmation du style roman. En liaison avec une exposition itinérante en Bourgogne (1999-2004) la réédition du remarquable ouvrage si bien documenté et illustré Les prémices de l'Art Roman en Bourgogne $\left({ }^{33}\right)$ assimile les recherches les plus récentes d'archéologie. Dans le long mûrissement de l'art roman, l'interdisciplinarité telle qu'elle est admirablement pratiquée à Auxerre permet de comprendre les relations qui existent entre les structures du paysage politique et culturel, la fonction des espaces liturgiques, les moyens et les techniques mises en œuvre et le décor.

Dans nos régions les reliques vont jouer un rôle moteur dans l'essor architectural. Les invasions normandes à la fin du $\mathrm{IX}^{\mathrm{e}}$ siècle obligent les religieux à quitter précipitamment leurs églises emportant leurs biens les plus précieux dans leur fuite devant l'envahisseur. De retour ils constatent les dégâts survenus et relèvent les édifices. Bien sûr il ne faut pas généraliser; d'autres circonstances peuvent intervenir: invasions hongroises au milieu du $\mathrm{X}^{\mathrm{e}}$ siècle, réformes monastiques... Autour de l'an mil la «blanche robe d'églises» évoquée par le chroniqueur clunisien Raoul Glaber prend en Basse-Lotharingie une coloration mosane, dans une orbite impériale très prégnante. Partout retentit le bruit des chantiers. Liège, capitale d'un diocèse et d'une principauté, donne l'exemple avec la reconstruction de la cathédrale Saint-Lambert par l'évêque Notger (972-1008), consacrée par son successeur Baldéric II en 1015 , avec les abbatiales bénédictines de Saint-Laurent et de Saint-Jacques et avec les sept collégiales, chacune améliorée ou même construite dans ce siècle. Une couronne d'églises enserre désormais la nouvelle Jérusalem: la «forteresse sacrée» liégeoise chère à Godefroid Kurth est debout pour des siècles. Avec Sainte-Croix au sommet du Publémont, la cathédrale SainteMarie où repose Lambert, le saint patron du diocèse, et Saint-Jean, inspirée d'Aix-la-Chapelle et sépulture du prélat, Notger érige à Liège un calvaire urbanistique digne des grandes cités de l'Empire: la croix, la Vierge et saint Jean.

La dévotion au saint local et à ses reliques motive les nouveaux aménagements architecturaux, pour accueillir le pèlerin et pour permettre le meilleur déroulement des cérémonies. C'est une cathédrale de type ottonien que Notger fit édifier à Liège : trois nefs, deux transepts, deux chœurs, l'un semi-circulaire à l'est et l'autre carré à l'ouest à l'emplacement exact du martyre de saint Lambert enserré dans une crypte révélée par les fouilles, périmètre

(33) D'Auxerre à Cluny, les premiers édifices après l'an mil, $2^{\mathrm{e}}$ édition revue et augmentée, Auxerre, Centre d'Etudes Médiévales, 2001. 
sacré soigneusement préservé intact jusque récemment. Des toutes les grandes églises mosanes du $\mathrm{XI}^{\mathrm{e}}$ siècle, étudiées par Luc-Francis Genicot, seule Nivelles, consacrée en 1046, est encore debout. Le chœur oriental rectangulaire surmonte une crypte-halle, véritable église semi-enterrée autour du culte de sainte Gertrude. A Huy, de la collégiale romane réédifiée en échange de la charte de franchise octroyée aux bourgeois par le princeévêque Théoduin de Bavière, et consacrée en 1066 en l'honneur de la Vierge et de saint Domitien, subsiste la vaste crypte à trois nefs terminées par une abside semi-circulaire. Le prélat y est enseveli.

Les fouilles archéologiques ont reconstitué d'autres grandes églises de type mosan, comme à Liège, à Stavelot ou à Malmedy. A Stavelot l'abbé Poppon inaugure sa prestigieuse abbatiale le 5 juin 1040 pour le saint patron fondateur Remacle: trois nefs, un transept avec des croisillons très développés et un chœur en abside semi-circulaire avec collatéral dont le retour s'effectuait sur les bras du transept. Ainsi les bas-côtés se prolongeaient autour du large transept et du sanctuaire en un déambulatoire qui permettait la circulation des pèlerins et libérait 1'édifice de toute affluence. Une vaste crypte à chevet plat, située hors d'œuvre à la suite et dans l'axe de l'abside, était semi-enfouie; au centre Poppon fit ériger son tombeau. L'accès se faisait par deux passages en pente douce, qui prenaient naissance dans le transept. Ce genre de crypte extérieure, typiquement mosane, se retrouve à Saint-Feuillen de Fosses, le seul exemple encore conservé, ou, d'après les sondages archéologiques, à Malmedy où Poppon s'est aussi avéré constructeur.

Fidèles à l'exemple carolingien, ces abbatiales ou collégiales de prestige dessinent un programme liturgique et hagiographique particulièrement révélateur des dévotions. Eglises de pèlerinage, elles organisent un dispositif circulatoire novateur, à la fois le déambulatoire et l'accès vers la crypte. Des édifices moyens ont survécu comme Celles, Hastière, ou Sclayn. Le transept est généralement de type bas - ses bras sont moins élevés que la nef centrale - et de part et d'autre un avant-corps occidental, et une crypte extérieure. A Celles une crypte se trouve aussi sous le massif occidental où une niche abritait jadis la châsse de saint Hadelin. A Ciney la crypte voûtée d'arêtes au XI $\mathrm{XI}^{\mathrm{e}}$ été prolongée au XII ${ }^{e}$ siècle. Parmi les très nombreuses églises rurales mosanes, aux tours massives si caractéristiques du paysage wallon, on n'a pas encore expliqué la fonction initiale de la petite crypte de Thynes près de Dinant $\left({ }^{34}\right)$.

Du côté scaldien, la construction de la collégiale de Soignies s'étale sur un siècle environ et cette église est un précieux témoin de l'évolution architecturale. Elle fut achevée vers 1120-1130. Le projet initial s'inspirait des modèles lotharingiens - Nivelles est proche. Les reliques du saint patron, saint Vincent, sont réservées au chœur mais primitivement sans accès déambulatoire pour les pèlerins. À Tournai, lieu d'un pèlerinage marial réputé depuis 1090, de la cathédrale romane, consacrée en 1171, subsiste la nef et le transept avec sa couronne de cinq tours achevées à l'époque gothique.

L'avant-nef des grandes églises est un espace d'accueil, intermédiaire entre le profane et le sacré. En pays mosan la formule la plus fréquente est un

(34) J.-L. JAVAUX et J. BUCHET, L'architecture romane en province de Namur, Namur, 1998, p. 107. 
massif occidental tripartite, dont les tours latérales sont des escaliers vers les étages. On accède notamment à une grande salle de réception dont le nom fait référence à l'empereur, mais qui peut aussi contenir les archives, la bibliothèque voire le trésor de la communauté. Enfin voici l'autel, souvent dédié à saint Michel, gardien du Paradis et peseur des âmes, qui évoque, à l'étage, son combat contre Satan. A la fonction militaire et défensive de l'avant-nef s'ajoute la fonction liturgique et symbolique ainsi que sépulcrale. Ces avant-nefs s'inscrivent dans la tradition des massifs occidentaux ottoniens, dont l'ampleur étonne, où les cloches et la lumière des bougies concourraient à marquer l'entrée solennelle dans le sacré. Alain Dierkens a magistralement tiré les conclusions du colloque d'Auxerre qui leur fut consacré, en insistant sur le caractère multifonctionnel de ces constructions en Europe $\left({ }^{35}\right)$. Les récentes campagnes archéologiques sur le site de l'ancienne avant-nef de l'abbaye Saint-Germain d'Auxerre ont permis de retrouver plusieurs états de l'occupation occidentale de ce site depuis le $\mathrm{V}^{\mathrm{e}}$ siècle. La mise au jour d'une avant-nef du IX $\mathrm{IX}^{\mathrm{e}}$ siècle, reconstruite sur un plan différent au début $\mathrm{du} \mathrm{XI} \mathrm{XI}^{\mathrm{e}}$ siècle, a conduit à renouveler la problématique. Les travaux de Jean-Charles Picard sur le quadriportique ou atrium des églises paléochrétiennes avaient déjà attiré l'attention sur les multiples fonctions de cet espace, entre accueil et recueillement, entre vie liturgique et lieu funéraire. Quelle filiation existe entre cette structure relativement bien datée et celle associée aussi bien aux grandes églises carolingiennes, connue sous le terme de "Westwerk», qu'à certaines églises romanes, comme les abbatiales bourguignonnes de Tournus ou de Vézelay? Les travaux archéologiques de ces quinze dernières années en France et dans plusieurs pays européens, pour certains encore inédits, les réflexions menées par divers chercheurs en séminaire ainsi que des thèses récemment soutenues ont motivé la tenue de ce très remarquable colloque international. Pour percevoir l'ampleur de ce paysage en diverses régions de France et d'Europe, la démarche est inscrite autant dans la longue durée que des approches croisées entre archéologie, textes et décors: une fois encore la pleine interdisciplinarité.

La protohistoire des œuvres d'art que sont les reliquaires nécessite chaque fois une recherche sur les reliques.

A l'initiative de Robert Didier et de Jacques Toussaint une exposition à Namur sur Hugo d'Oignies a retracé l'activité d'orfèvre et d'enlumineur vers 1228-1240 de ce religieux, originaire de Walcourt $\left({ }^{36}\right)$. A Oignies, petit établissement religieux de la basse Sambre, Hugo exerce son talent et réalise un trésor dont les Sœurs de Notre-Dame à Namur sont aujourd'hui les fidèles gardiennes. Le cardinal Jacques de Vitry $(\dagger 1240)$, riche mécène, fut un généreux «fou de reliques»: il en procura en abondance au jeune prieuré d'Oignies. Par ses dons, il favorisa l'éclosion du talent de l'artiste. Plusieurs reliquaires sont anthropomorphiques: côte de saint Pierre, pied de saint Jacques, et pied de saint Blaise. Saint-Materne de Walcourt conserve une croix- reliquaire fleu-

(35) Chr. SAPIN, ed., Avant-nefs et espaces d'accueil dans l'église entre le IVe et le XII siècle. Actes du colloque international du CNRS à Auxerre (1999) organisé par le Centre d'Etudes Médiévales d'Auxerre et l'Université de Bourgogne, Auxerre, 2002.

(36) Autour de Hugo d'Oignies, Namur, 2003. 
ronnée à double traverse (Atelier d'Oignies, ca. 1250-1260), la plus grande qui soit conservée en Wallonie, somptueuse par ses pierres et ses nielles, par ses filigranes en rinceaux au décor très varié et d'une virtuosité technique remarquable; on y découvre aussi un reliquaire- tourelle de la Vraie-Croix unique en son genre. Un colloque organisé conjointement à l'exposition apporta des informations complémentaires sur Hugo et déborda plus largement sur le cadre géographique et chronologique : Jerzy Pietrusinski, Les rapports artistiques entre la région mosane et la Pologne aux XII ${ }^{e}-X I I I^{e}$ siècles; Peter Kurmann, Reims et Bamberg: un exemple du transfert de style à l'époque de Hugo d'Oignies; Elisabeth Taburet-Delahaye, Le bras-reliquaire de saint Landelin conservé à Crespin (nord France); Javier Martinez de Aguirre, Le reliquaire du Saint Sépulcre de Pampelune; Claudine Lautier, Le vitrail de Charlemagne à Chartres et les reliques du trésor de la cathédrale; Anna Rosa Calderoni Masetti, Plaquettes cousues sur la "ceinture» de la cathédrale de Pise attribuées au cercle de Nicolas de Pise aux environs de 1260, et Angela Franco Mata, L'iconographie dans les objets liturgiques $\left({ }^{37}\right)$. Des aspects techniques de l'orfèvrerie médiévale furent également traités. La couronnereliquaire des Saintes Épines du Trésor de la cathédrale Saint-Aubain à Namur a fait l'objet d'une intéressante étude de Winfried Wilhelmy, interpellante par rapport à la notice du catalogue. Quelle chance de voir ainsi reprendre par un spécialiste le dossier complet d'une ouvre de tout premier plan! Non seulement pour faire le point - on n'insistera jamais assez sur l'acquis de l'érudition du $\mathrm{XIX}^{\mathrm{e}}$ siècle - mais aussi pour intégrer les progrès de la recherche et souvent apporter du neuf. L'initiative sur Hugo d'Oignies en est un bel exemple, comme la châsse de Nivelles précédemment, ou, du même Robert Didier, le béryl de Lothaire qui, paru dans une collection confidentielle $\left({ }^{38}\right)$, s'inscrit dans cet intérêt actuel manifesté pour la glyptique $\left({ }^{39}\right)$.

La châsse actuelle de saint Remacle, celle du XIII ${ }^{\mathrm{e}}$ siècle (1263-1268) toujours conservée à Stavelot, n'avait pas encore fait l'objet d'une contextualisation historique, pourtant bien nécessaire à sa compréhension qu'elle soit historique ou artistique $\left({ }^{40}\right)$. On peut en dire autant de «la Vierge telle que saint Luc l'aurait peinte", l'une des reliques majeures de l'ancienne cathédrale SaintLambert de Liège, ramenée de Constantinople et qui vient de participer à une exposition sur l'art byzantin de la fin du Moyen Âge $\left({ }^{41}\right)$. Cette œuvre a pu bénéficier d'une étude d'archéométrie complète dont les résultats sont annoncés.

Les récents travaux de restauration de la collégiale Saint-Martin de Candes ont permis la découverte de reliques sous le maître-autel placé en

(37) Dans le Bolletino del Museo Arqueologico Nacional de Madrid (t. XV, 1997, p. 175-196), Angela Franco Mata a publié le "Mobiliario Medieval en el Museo Arqueologico Nacional (Siglos VIII al XV)".

(38) R. DIDIER, "A propos du «béryl» de Lothaire et d'orfèvrerie des XIIc et XIIIc siècles provenant de l'ancienne abbaye de Waulsort", dans Notes waulsortoises rassemblées par A. WAYENS, t. V, Waulsort, 1987, p. 207-251.

(39) G. KORNBlUTH, Engraved Gems of the Carolingian Empire, Pennsylvanie, University Park, 1995.

(40) Ph. GEORGE, "La châsse de saint Remacle (1263-1268) et Liège", dans Bulletin de la Société Royale Le Vieux-Liège, t. XIV, $\mathrm{n}^{\circ}$ 298-299, 2002, p. 317-334. 
$1783\left({ }^{42}\right)$. On rappellera, si besoin en est, que c'est à Candes en Anjou que le treizième apôtre rendit l'âme. Son corps fut emporté de nuit par les Tourangeaux et ramené à Tours. Quatre ardoises $(1729,1783,1852-1856)$ furent découvertes et une fiole en verre avec de la terre «où est mort st Martin sur la cendre et le cilice» et de saint Maurice. Le souvenir de la visite de Martin à Agaune a été perpétué à travers les siècles depuis la correspondance de Guibert-Martin de Gembloux jusqu'aux oeuvres d'art comme la remarquable tapisserie du Trésor d'Angers, où le miracle est représenté : “COMMENT MONSEIGNEUR SAINCT MARTIN FIST RENDRE A LA TERRE LE SANG DE SAINCT MAURICE ET DE SES COMPAIGNONS" $\left({ }^{43}\right)$.

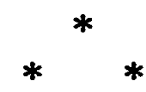

Le mot «reliques» a-t-il enfin perdu toute connotation péjorative? On aimerait le penser. Les reliques, nouvel objet historique, poursuivent leur carrière scientifique. Leurs liens avec les oeuvres d'art nous ont incité à leur consacrer un ouvrage fait d'un cheminement historique doublé d'une analyse d'archéologie et d'histoire de l'art sur l'espace mosan $\left({ }^{44}\right)$. Dans son bel ouvrage sur l'image de l'évêque dans le pontifical au Moyen Âge, Eric Palazzo aborde la dédicace de l'église et la consécration d'autel $\left({ }^{45}\right)$. Son abondante iconographie nous met à l'esprit un autre thème à aborder à propos des reliques: leur iconographie. Les exemples ne sont pas légion; rappelons la Vie de saint Hubert d'Hubert le Prévôt illustrée par Loyset Liédet (1463), la dévotion à saint Agilolf sur son retable à Cologne (1521), ou, plus anciens, le magnifique manuscrit de la Bibliothèque Municipale de Tours qui montre l'élévation du chef de saint Martin en 1317, l'antependium du XIV ${ }^{\mathrm{e}}$ siècle de Saint-Martin de Liège la Subventio et Reversio Martini $\left({ }^{46}\right)$, et pour revenir à Fleury le portail nord consacré à la découverte des reliques de saint Benoît: un moine puise dans le sarcophage pour en extraire les ossements recueillis par un autre moine dans un panier....

La richesse et l'extrême variété des thèmes perçus à travers cet aperçu bibliographique général récent prouvent tout le chemin parcouru. Mais les horizons sont immenses. La recherche n'en est rendue que plus excitante.

(41) Ph. GEORGE, "Icon with the Virgin Hodegetria", dans Catalogue de l'exposition H. C. EVANS, ed., Byzantium. Faith and Power (1261-1557), New York, The Metropolitan Museum, 2004,658 p. in $-4^{\circ}, n^{\circ} 150$ p. $252-253$.

(42) Renseignements très aimablement transmis par notre collègue Guy du Chazaud, Conservateur des Antiquités et Objets d'Art d'Indre-et-Loire, et son collaborateur Mr. René-Charles Guilbaud à la suite de notre visite. Nous les en remercions très vivement.

(43) G. MASSIN-LEGOFF et Et. VACQUET, Tapisseries d'Anjou (XVe-XVIII ${ }^{e}$ siècles) au Trésor de Liège, Liège, 2004, p. 62-65.

(44) Ph. GEORGE, Reliques et arts précieux en pays mosan. Du Haut Moyen Âge à l'époque contemporaine, Liège, 2002.

(45) E. PAlazzo, L'évêque et son image. L'illustration du Pontifical au Moyen Âge, Turnhout, Brepols, 1999, p. 307-356.

(46) Catalogue de l'exposition Saint-Martin, mémoire de Liège, Liège, 1990, p. 100 et 101. 